\title{
The Effect of Fibular Reposition Taping on Postural Control in Individuals With Chronic Ankle Instability: A Critically Appraised Topic
}

\author{
Bradley C. Jackson, Robert T. Medina, Stephanie H. Clines, Julie M. Cavallario, \\ and Matthew C. Hoch
}

\begin{abstract}
Clinical Scenario: History of acute ankle sprains can result in chronic ankle instability (CAI). Arthrokinematic changes resulting from CAI may restrict range of motion and contribute to postural control deficits. Mulligan or fibular reposition taping (FRT) has been suggested as a means to realign fibular positional faults and may be an effective way to improve postural control and balance in patients with CAI. Clinical Question: Is there evidence to suggest that FRT will improve postural control for patients with CAI in the affected limb compared with no taping? Summary of Key Findings: Three of the 4 included studies found no significant difference in postural control in patients receiving FRT compared with sham or no tape. Clinical Bottom Line: There is moderate evidence refuting the use of FRT to improve postural control in patients with CAI. Strength of Recommendation: There is grade B evidence to support that FRT does not improve postural control in people with CAI.
\end{abstract}

Keywords: Mulligan tape, ankle sprain, balance

\section{Clinical Scenario}

Ankle sprains are a common injury affecting both athletes ${ }^{1}$ and the general population. ${ }^{2}$ Physical deficits such as mechanical instability, sensorimotor impairment, and recurrent pain can persist after acute symptoms have resolved ${ }^{3}$ and lead to a decrease in physical activity and health-related quality of life. ${ }^{4,5}$ Chronic ankle instability (CAI) is a common residual problem resulting from lateral ankle sprains characterized by sensations of joint instability, episodes of "giving way" during activities of daily living, mechanical laxity, ${ }^{6}$ and diminished postural control or balance. ${ }^{7}$ Postural control impairments may be associated with alterations in arthrokinematics, which occur as a result of joint trauma. ${ }^{8}$ Although other factors may also contribute to alterations in postural control, this paper will focus on the influence of arthrokinematic alterations following acute ankle sprains. Arthrokinematic restrictions resulting from lateral ankle sprains reduce the ability to achieve full physiological range of motion of the joint, ${ }^{9}$ which has been demonstrated to negatively impact postural control. ${ }^{10,11}$ One such arthrokinematic alteration associated with inversion ankle sprains is an anterior and inferior shift of the fibula relative to the talus. Positional faults of the fibula are thought to limit accessory motions in the ankle, which may result in hypomobility ${ }^{12}$ and negatively affect sensorimotor function. ${ }^{13,14}$ To address fibular positioning faults, it has been suggested that fibular reposition taping (FRT) may improve postural control in patients with CAI by mechanically realigning the fibula and restore preinjury arthrokinematics. ${ }^{15-19}$ Therefore, FRT could become a useful tool to aid clinicians in the treatment of patients with CAI and lead to reduced symptoms and improved performance during physical activity.

Jackson, Medina, Clines, and Cavallario are with Old Dominion University, Norfolk, VA. Hoch is with the University of Kentucky, Lexington, KY. Clines (sclin001@ odu. edu) is corresponding author.

\section{Focused Clinical Question}

Is there evidence to suggest that FRT will improve postural control for patients with CAI in the affected limb compared with no taping?

\section{Summary of Search, "Best Evidence" Appraised, and Key Findings}

- The literature was searched for studies of level 3 evidence or higher that investigated the effect of FRT on postural control in patients with CAI.

- The search of the literature yielded 7 possible studies for inclusion.

- Four studies ${ }^{16-19}$ met the inclusion criteria and were critically appraised using the 16-item Downs and Black checklist ${ }^{20}$ in which individual studies were categorized as low $(<60 \%)$, moderate $(60 \%-74.9 \%)$, or high $(>75 \%)$ quality based on the number of identified criteria.

- Three of the studies ${ }^{16-18}$ suggested that FRT does not improve postural control in individuals with CAI.

- Whereas 1 study $^{19}$ indicated significant improvements in postural control in individuals with CAI.

\section{Clinical Bottom Line}

There is moderate evidence refuting the use of FRT to improve postural control in patients with CAI.

\section{Strength of Recommendation}

There is grade B evidence that FRT does not improve postural control in people with CAI. The Centre of Evidence-Based Medicine (CEBM) Levels of Evidence 2011 recommends grade B for a combination of level 1-3 evidence with consistent findings. 


\section{Search Strategy}

\section{Terms Used to Guide Search Strategy}

- Patient group: chronic ankle instability

- Intervention: Mulligan OR fibular reposition taping

- Comparison: no tape OR sham

- Outcome: postural control OR balance

\section{Sources of Evidence Searched}

- EBSCOhost

- SPORTDiscus

- CINAHL

- PubMed

\section{Inclusion and Exclusion Criteria}

\section{Inclusion Criteria}

- Studies that compared a FRT condition to a no tape or sham condition in people with CAI

- Studies that included a measure of postural control as an outcome measure

- Level 3 evidence or higher

- Limited to English language

- Limited to humans

- Limited to the past 10 years

\section{Exclusion Criteria}

- Studies that did not include subjects with CAI

- Studies that did not include a FRT condition

- Studies that did not include postural control as an outcome measure

\section{Results of Search}

Four relevant studies ${ }^{16-19}$ were obtained and are categorized in Table 1 (based from Levels of Evidence, CEBM, 2011). ${ }^{21}$

\section{Best Evidence}

The studies in Table 2 were identified as best evidence and therefore selected for inclusion in this critically appraised topic. These studies were included because they were categorized as level 3 evidence or higher and examined the effect of FRT on postural control compared with no tape or sham tape in individuals presenting with CAI.

\section{Implications for Practice, Education, and Future Research}

Only one investigation ${ }^{19}$ identified significant changes in postural control following FRT application, with the remaining studies concluding there were no changes in static or dynamic postural control following FRT application. ${ }^{16-18}$ Based on the consistency of the findings and level of evidence, a grade B strength of recommendation was made as there is moderate evidence to refute using FRT as an intervention to enhance postural control in patients with CAI.

The method of assessing postural control did not appear to be an important factor in FRT outcomes. Most of the included studies utilized the Star Excursion Balance Test to assess dynamic postural control, while a single study ${ }^{16}$ utilized a force plate to assess singlelimb static postural control. The method of assessing postural control may be an important consideration when attempting to identify changes in postural control following an intervention in patients with CAI. This point is supported by a critically appraised topic $^{22}$ that identified postural control impairments following short foot exercises when assessed with dynamic measures but not with static measures in those with CAI. However, talocrural joint mobilization resulted in immediate improvements in static balance but not dynamic balance in those with CAI. ${ }^{23}$ Therefore, the identification of postural control improvements following intervention may be directly related to the type and theorized treatment effect of the intervention under investigation. In the case of FRT, only one of the studies ${ }^{17-19}$ that measured dynamic postural control identified a significant improvement, while the single study ${ }^{16}$ that measured static balance also did not identify improvement. The study by Someeh et $\mathrm{al}^{19}$ identified reach distance improvements of $4 \%$ to $5 \%$ associated with effect sizes ranging from moderate to large $(0.60-0.75)$. Although this study did have the greatest quality index score, the consistency of the findings from the other included studies indicate that FRT may not improve postural control in those with CAI regardless of the measurement technique. Based on these results, future studies that further pursue this line of inquiry should give careful consideration to selecting postural control measures, which best assess the theorized benefits of FRT in those with CAI.

The application of the FRT intervention among the 4 studies $^{16-19}$ was very similar (Table 2). Three of the studies used tape directly on the skin starting at the distal malleolus of the fibula. ${ }^{16,17,19}$ However, one investigation ${ }^{18}$ utilized the same taping technique with the addition of cover roll between participant's skin and the leukotape but still did not alter postural control measurements. ${ }^{18}$ Overall, a homogenous FRT technique was applied across all four studies. ${ }^{16-19}$ However, none of the studies assessed fibular position at any time

Table 1 Summary of Study Designs and Level of Evidence Based on CEBM 2011

\begin{tabular}{llcc}
\hline Level of evidence & \multicolumn{1}{c}{ Study design } & Number located & References \\
\hline 2 & Crossover & 1 & Wheeler et al $^{18}$ \\
2 & One-way repeated measures & 3 & Hopper et al $^{16}$ \\
& & Delahunt et al $^{17}$ \\
& & Someeh et al $^{19}$ \\
\hline
\end{tabular}

Abbreviation: CEBM, Centre of Evidence-Based Medicine. 


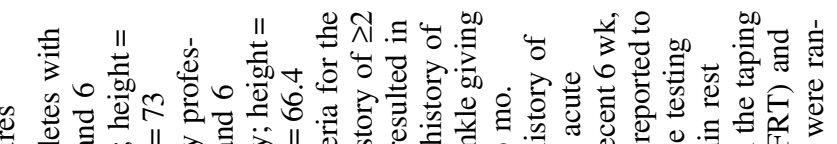

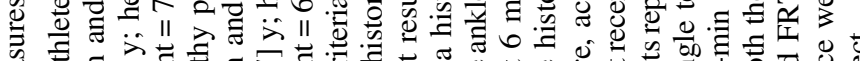

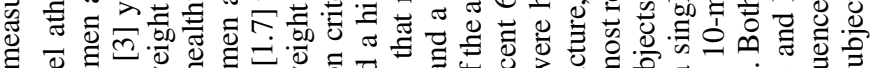

ठ

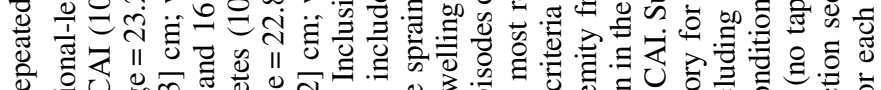

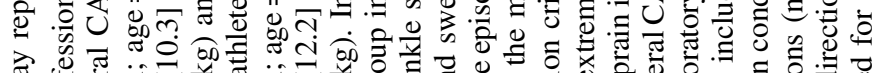

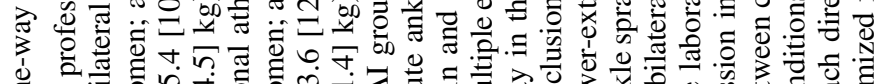

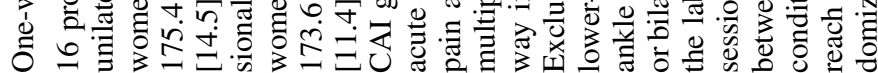

に

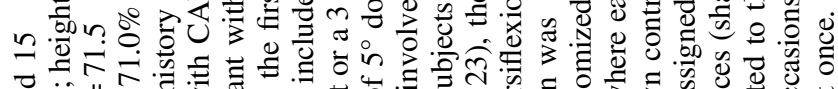

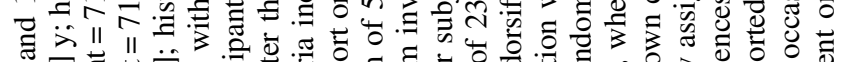

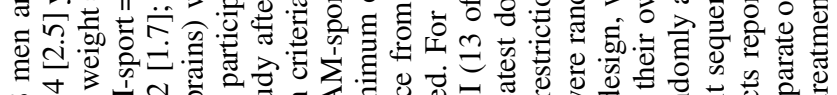

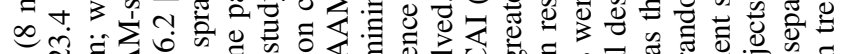

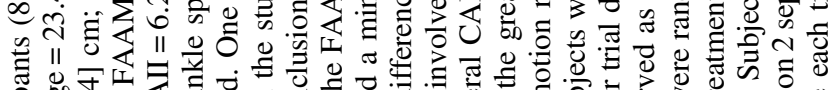

$\frac{\pi}{2}$

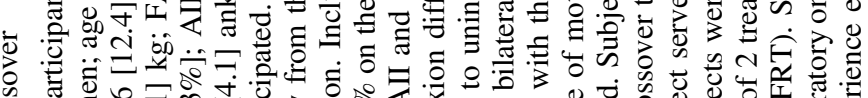

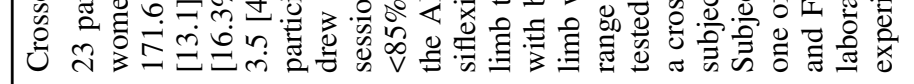

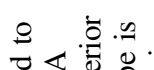

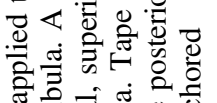

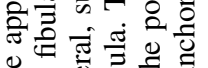

赵记

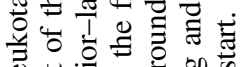

ब

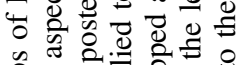

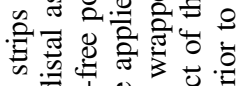

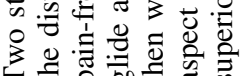

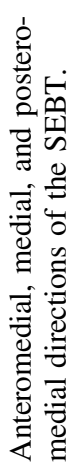

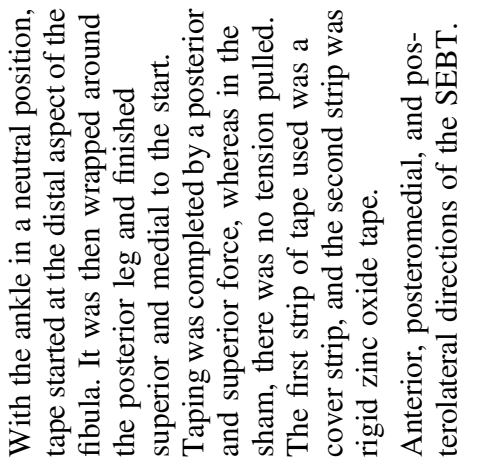

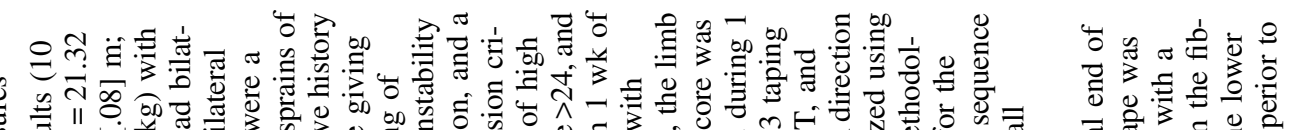

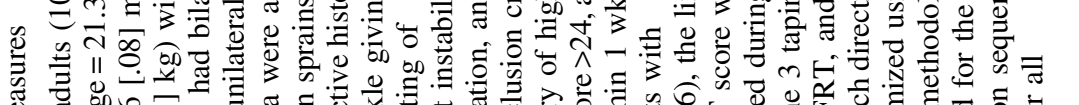

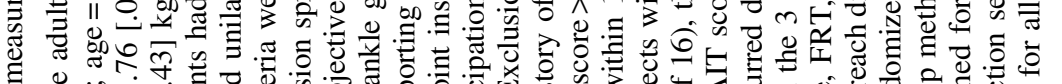

节

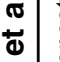

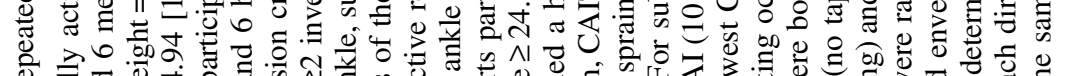

竞

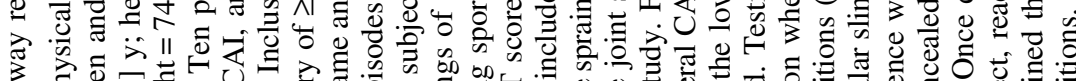

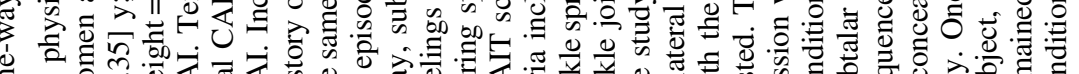

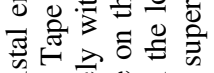

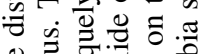

음

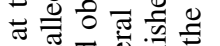

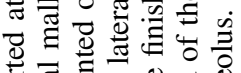

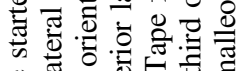

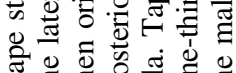

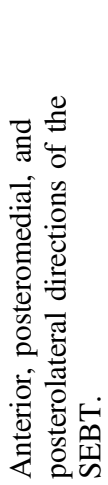

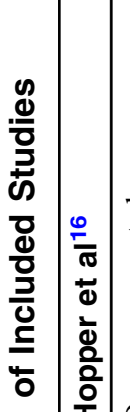

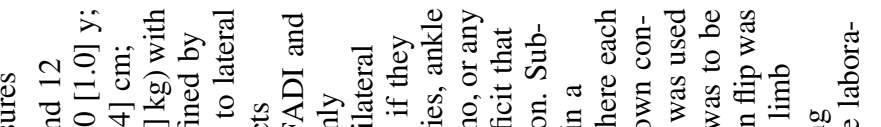

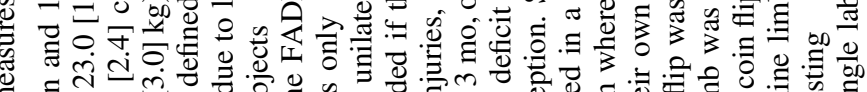

过

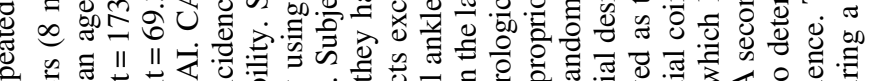

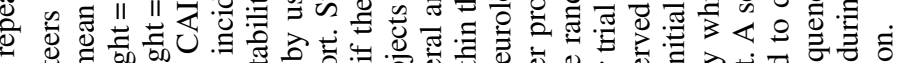

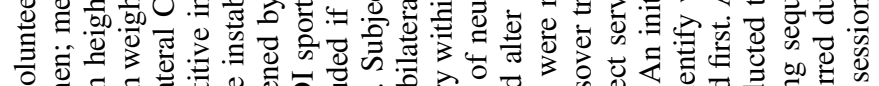

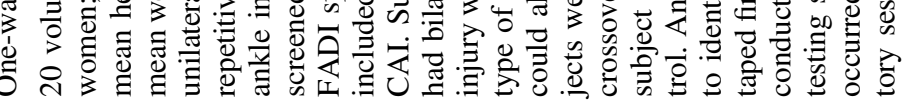

$\stackrel{8}{8}$

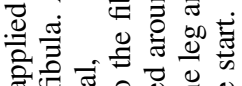

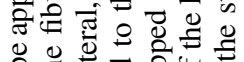

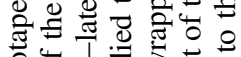

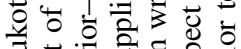

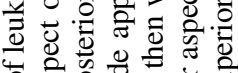

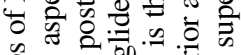

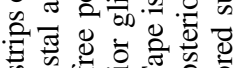

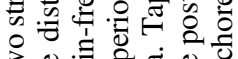

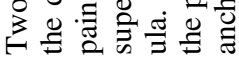

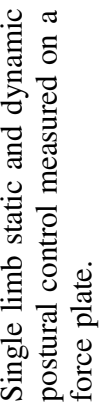

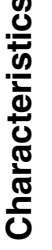

商

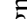

कू⿻

气ั๊

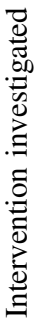

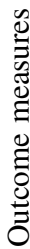




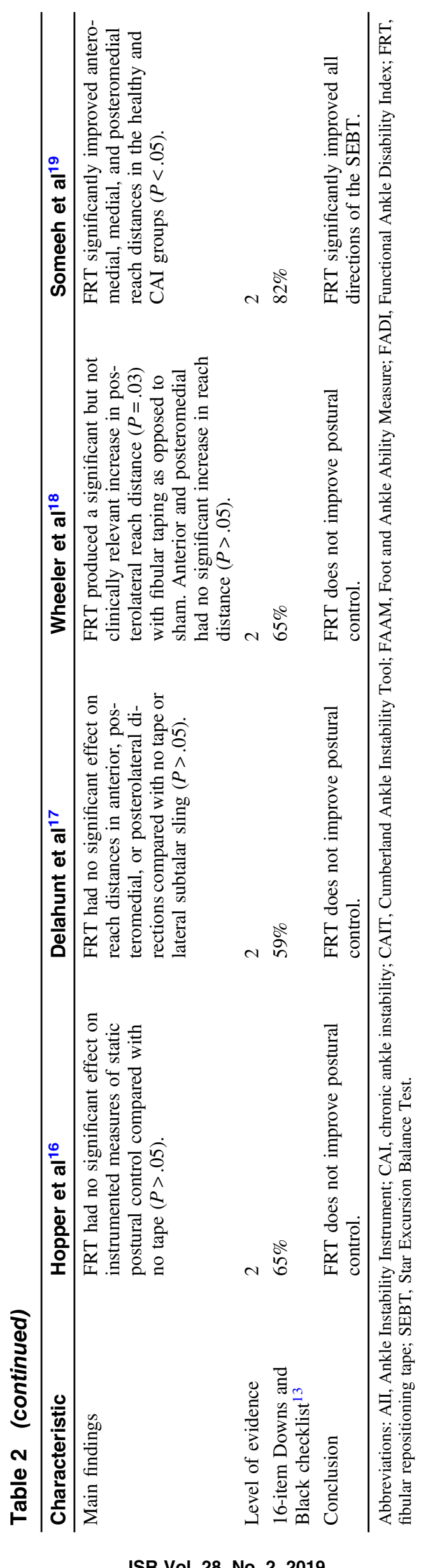


point during the investigation. ${ }^{16-19}$ Whether subjects included in these investigations actually had a positional fault is unclear and therefore brings to question whether the FRT intervention achieved the intended purpose of mechanically correcting the patients fibular position and arthrokinematics at the ankle. As a result, it is possible that the FRT intervention was not indicated in these subjects or that the applied intervention was not achieving the intended purpose of mechanically addressing the positional fault. Both factors may have impacted the results of these studies contributing to a lack of significant findings. Future studies should consider examining FRT in patients who exhibit a confirmed malpositioning of the distal fibula and the concurrent mechanical effects to further understand the role of this treatment in the management of patients with CAI.

The use of FRT in patients with CAI has also been explored in the context of motor neuron excitability, which may play a role in postural control. ${ }^{24}$ Current literature provides inconclusive results regarding the ability of FRT to provide clinically significant neuromuscular changes in patients with $\mathrm{CAI}$ as recent investigations have identified both significant changes ${ }^{25}$ and no changes ${ }^{26}$ in neuromotor excitability in this population. None of the investigations reviewed for this paper included any neuromuscular outcome measures and discrepancies in the FRT literature in both areas of motor neuron excitability and postural control are present. This highlights the complexity of CAI and the need for a multidimensional approach to treatment of this condition.

The included articles were critically appraised using the 16item Downs and Black checklist, ${ }^{20}$ which identified 1 low-quality, 2 moderate-quality, and 1 high-quality study (Table 2). Commonly missed items included recruitment over the same time period, description of confounders, adjustments for confounding in the analysis, and participants representative of the population. ${ }^{20}$ Based on the consistent findings from level 2 evidence based on the CEBM 2011 guidelines, a grade B strength of recommendation was made as there is moderate evidence to refute using FRT as an intervention to enhance postural control in patients with CAI. All studies were categorized as level 2 because they utilized an experimental design that contained randomization and a control condition. It should be noted that the study by Wheeler et al ${ }^{18}$ had a stronger design as it met the criteria of a crossover trial as subjects were randomized to a treatment order on different days, which created a true washout period between conditions. Although we categorized the included studies as a relatively high level of evidence, no randomized controlled trials were identified, which would be considered the gold standard experimental design for assessing therapeutic interventions. Based on the brevity of the FRT intervention in the included studies, the implemented designs are certainly appropriate and do not appear to have created bias based on the limited number of identified improvements. This critically appraised topic should be reviewed in 2 years or when additional best evidence becomes available, which may change the clinical bottom line for this clinical question.

\section{References}

1. Fong DT, Hong Y, Chan LK, Yung PS, Chan KM. A systematic review on ankle injury and ankle sprain in sports. Sports Med. 2007;37(1):73-94. PubMed ID: 17190537 doi:10.2165/00007256200737010-00006

2. Bridgman SA, Clement D, Downing A, Walley G, Phair I, Maffulli N. Population based epidemiology of ankle sprains attending accident and emergency units in the west midlands of england, and a survey of UK practice for severe ankle sprains. Emerg Med J. 2003;20(6): 508-510. PubMed ID: 14623833 doi:10.1136/emj.20.6.508

3. van Rijn RM, van Os AG, Bernsen RM, Luijsterburg PA, Koes BW, Bierma-Zeinstra SM. What is the clinical course of acute ankle sprains? A systematic literature review. Am J Med. 2008;121(4): 324-331.e7. PubMed ID: 18374692 doi:10.1016/j.amjmed.2007. 11.018

4. Houston MN, Hoch JM, Hoch MC. Patient-reported outcome measures in individuals with chronic ankle instability: a systematic review. J Athl Train. 2015;50(10):1019-1033. PubMed ID: 26332028 doi:10.4085/1062-6050-50.9.01

5. Hubbard-Turner T, Turner MJ. Physical activity levels in college students with chronic ankle instability. J Athl Train. 2015;50(7): 742-747. PubMed ID: 25898110 doi:10.4085/1062-6050-50. 3.05

6. Gribble PA, Delahunt E, Bleakley C, et al. Selection criteria for patients with chronic ankle instability in controlled research: a position statement of the International Ankle Consortium. Br J Sports Med. 2014;48(13):1014-1018. PubMed ID: 24255768 doi:10.1136/ bjsports-2013-093175

7. Arnold BL, De La Motte S, Linens S, Ross SE. Ankle instability is associated with balance impairments: a meta-analysis. Med Sci Sport Exer. 2009;41(5):1048-1062. doi:10.1249/MSS.0b013e318192d044

8. Hoch MC, Staton GS, McKeon PO. Dorsiflexion range of motion significantly influences dynamic balance. J Sci Med Sport. 2011;14(1):90-92. PubMed ID: 20843744 doi:10.1016/j.jsams. 2010.08.001

9. Terada M, Pietrosimone BG, Gribble PA. Therapeutic interventions for increasing ankle dorsiflexion after ankle sprain: a systematic review. J Athl Train. 2013;48(5):696-709. PubMed ID: 23914912 doi:10.4085/1062-6050-48.4.11

10. Cruz-Díaz D, Lomas Vega R, Osuna-Pérez MC, Hita-Contreras F, Martínez-Amat A. Effects of joint mobilization on chronic ankle instability: a randomized controlled trial. Disabil Rehabil. 2015;37(7):601-610. doi:10.3109/09638288.2014.935877

11. Basnett CR, Hanish MJ, Wheeler JT, et al. Ankle dorsiflexion range of motion influences dynalic balance in individuals with chronic ankle instability. Sports Phys Ther. 2013;8(2):121-128.

12. Denegar CR, Miller SJ 3rd. Can chronic ankle instability be prevented? Rethinking management of lateral ankle sprains. J Athl Train. 2002;37:430-435. PubMed ID: 12937564

13. Hubbard TJ, Hertel J, Sherbondy P. Fibular position in individuals with self-reported chronic ankle instability. J Orthop Sports Phys Ther. 2006;36(1):3-9. PubMed ID: 16494068 doi:10.2519/jospt. 2006.36.1.3

14. Hubbard TJ, Hertel J. Anterior positional fault of the fibula after subacute lateral ankle sprains. Man Ther. 2008;13(1):63-67. PubMed ID: 17188923 doi:10.1016/j.math.2006.09.008

15. Sailors ME, Mulligan BR. Manual Therapy: NAGS, SNAGS, MWMS, Etc. 3rd ed. Wellington, New Zealand: Plane View Services Ltd.; 1995.

16. Hopper D, Samsson K, Hulenik T, Ng C, Hall T, Robinson K. The influence of Mulligan ankle taping during balance performance in subjects with unilateral chronic ankle instability. Phys Ther Sport. 2009;10(4):125-130. PubMed ID: 19897165 doi:10.1016/j.ptsp. 2009.07.005

17. Delahunt E, McGrath A, Doran N, Coughlan GF. Effect of taping on actual and perceived dynamic postural stability in persons with chronic ankle instability. Arch Phys Med Rehabil. 2010; 91(9):1383-1389. PubMed ID: 20801256 doi:10.1016/j.apmr. 2010.06 .023 
18. Wheeler TJ, Basnett CR, Hanish MJ, et al. Fibular taping does not influence ankle dorsiflexion range of motion or balance measures in individuals with chronic ankle instability. J Sci Med Sport. 2013;16(6):488-492. PubMed ID: 23537695 doi:10.1016/j.jsams. 2013.02.012

19. Someeh M, Norasteh AA, Daneshmandi H, Asadi A. Immediate effects of Mulligan's fibular repositioning taping on postural control in athletes with and without chronic ankle instability. Phys Ther Sport. 2015;16(2):135-139. PubMed ID: 25165014 doi:10.1016/j. ptsp.2014.08.003

20. Downs SH, Black N. The feasibility of creating a checklist for the assessment of the methodological quality both of randomised and non-randomised studies of health care interventions. J Epidemiol Community Health. 1998;52(6):377-384. PubMed ID: 9764259 doi:10.1136/jech.52.6.377

21. OCEBM Levels of Evidence Working Group. The oxford 2011 levels of evidence. Oxford centre for evidence-based medicine website. http://www.cebm.net/index.aspx?o=5653. Updated March 2009. Accessed July 11, 2018.
22. Mignogna C, Welsch LA, Hoch MC. The effects of short foot exercises on postural control: a critically appraised topic. Int $J$ Athl Ther Train. 2016;21(6):8-12. doi:10.1123/ijatt.2016-0049

23. Hoch MC, McKeon PO. Joint mobilization improves spatiotemporal postural control and range of motion in those with chronic ankle instability. J Orthopaedic Research. 2011;29(3):326-332. doi:10. 1002/jor.21256

24. Wikstrom EA, Fournier KA, McKeon PO. Postural control differs between those with and without chronic ankle instability. Gait Posture. 2010;32(1):82-86. PubMed ID: 20418101 doi:10.1016/j. gaitpost.2010.03.015

25. Chou E, Kim KM, Baker AG, Hertel J, Hart JM. Lower leg neuromuscular changes following fibular reposition taping in individuals with chronic ankle instability. Man Ther. 2013;18(4): 316-320. PubMed ID: 23302515 doi:10.1016/j.math.2012.11.004

26. Grindstaff TL, Hanish MJ, Wheeler TJ, et al. Fibular taping does not alter lower extremity spinal reflex excitability in individuals with chronic ankle instability. J Electromyogr Kinesiol. 2015;25(2):253-259. PubMed ID: 25727519 doi:10.1016/j.jelekin.2015.01.009 